\title{
PSEUDO-CONFORMAL GEOMETRY: FUNCTIONS OF TWO COMPLEX VARIABLES
}

\author{
EDWARD KASNER AND JOHN DECICCO
}

1. Introduction. The theory of functions of a single complex variable is essentially identical with the conformal geometry of the real (or complex) plane. However, this is not the case in the theory of functions of two independent complex variables. Any pair of functions of two complex variables induces a correspondence between the points of a real (or complex) four-dimensional space $S_{4}$. The infinite group $G$ of all such correspondences is obviously not the conformal group of $S_{4}$. Poincaré in his fundamental paper in Palermo Rendiconti (1907) has called $G$ the group of regular transformations. In an abstract presented to the Society, 1908, Kasner found it more appropriate to term it the pseudo-conformal group $G$.

In a preceding paper, Kasner has given a purely geometric characterization. His main result is that the pseudo-conformal group $G$ is characterized by the fact that it leaves invariant the pseudo-angle between any curve and any hypersurface at their point of intersection. ${ }^{1}$

In the present work, we shall find all the differential invariants of first order between the curves, surfaces, and hypersurfaces at a given point under the pseudo-conformal group. We shall take every combination of any two elements - six possible cases. ${ }^{2}$ The number of independent invariants may be 0,1 , or 2 .

A general pair of curve elements possesses no invariants. However, in the special case of an isoclinal pair, there is a unique invariant (the angle between them). A similar result is true for two hypersurface elements.

A hypersurface element and a curve element possess only one invariant-the pseudo-angle. ${ }^{1}$

To any general surface element $S$, there is associated a quadric regulus $R$ of curve elements. There are no invariants between a general surface element $S$ and a curve element $e$ which is not on the regulus $R$ of $S$. On the other hand, if $e$ is in $R$, then there is a unique

Presented to the Society, September 5, 1941; received by the editors July 19, 1941.

${ }^{1}$ Kasner, Conformality in connection with functions of two complex variables, Transactions of this Society, vol. 48 (1940), pp. 50-62. See also the following paper: Kasner, Biharmonic functions and certain generalizations, American Journal of Mathematics, vol. 58 (1936), pp. 377-390.

${ }^{2}$ We sha!l denote by $e$ a curve element, that is, a lineal element; by $S$ a general surface element; and by $\pi$ a hypersurface element. The six possible cases are $(e, e)$, $(e, \pi),(\pi, \pi),(e, S),(\pi, S),(S, S)$. 
invariant between $e$ and $S$. We find similar results by substituting hypersurface element $\pi$ for curve element $e$ in the preceding statements.

Finally, two general surface elements possess two independent invariants.

2. The minimal coordinates. Let $(\epsilon, \eta, \zeta, \xi)$ denote the cartesian coordinates of any point of complex euclidean four-space $S_{4}$. In our work, we shall find it very convenient to introduce the minimal coordinates $(x, y, u, v)$ defined by

$$
x=\epsilon+i \eta, \quad y=\zeta+i \xi, \quad u=\epsilon-i \eta, \quad v=\zeta-i \xi .
$$

The inverse of this correspondence is

(2) $\epsilon=\frac{1}{2}(x+u), \eta=\frac{1}{2 i}(x-u), \zeta=\frac{1}{2}(y+v), \xi=\frac{1}{2 i}(y-v)$.

In minimal coordinates, the distance $d s$ between any two nearby points of space is

$$
d s^{2}=d x d u+d y d v \text {. }
$$

If $\theta$ is the angle between any two directions of space, then

$$
\cos \theta=\frac{d x_{1} d u_{2}+d x_{2} d u_{1}+d y_{1} d v_{2}+d y_{2} d v_{1}}{2\left[\left(d x_{1} d u_{1}+d y_{1} d v_{1}\right)\left(d x_{2} d u_{2}+d y_{2} d v_{2}\right)\right]^{1 / 2}} .
$$

3. The pseudo-conformal group This is given in minimal coordinates by

(5) $X=X(x, y), \quad Y=Y(x, y), \quad U=U(u, v), \quad V=V(u, v)$,

where $X_{x} Y_{y}-X_{y} Y_{x} \neq 0$ and $U_{u} V_{v}-U_{v} V_{u} \neq 0$. Our problem is to inaugurate the study of the geometry of this group. ${ }^{3}$

In what follows, we shall omit from consideration the special minimal planes $x=$ const., $y=$ const., and $u=$ const., $v=$ const. Obviously our pseudo-conformal group may be defined as that preserving the $2 \infty^{2}$ special minimal planes.

\section{The pseudo-conformal geometry of differential elements of first} order. In this and the following sections, we shall mainly be interested in the geometry of the curve elements at a fixed point of $S_{4}$. Obviously these form a three-dimensional manifold $\Sigma_{3}$.

${ }^{3}$ We shall study only the continuous pseudo-conformal group, not the more extensive mixed group obtained by adjoining functions of the conjugate complex variables. 
The pseudo-conformal group induces the seven-parameter group $G_{7}$ among the $\infty^{3}$ curve elements of $\Sigma_{3}$ at a given point, defined as follows

$$
\begin{array}{ll}
\rho X^{\prime}=a x^{\prime}+b y^{\prime}, & \rho U^{\prime}=\alpha u^{\prime}+\beta v^{\prime}, \\
\rho Y^{\prime}=c x^{\prime}+d y^{\prime}, & \rho V^{\prime}=\gamma u^{\prime}+\delta v^{\prime},
\end{array}
$$

where $a d-b c \neq 0$ and $\alpha \delta-\beta \gamma \neq 0$. Note that $\left(\rho x^{\prime}, \rho y^{\prime}, \rho u^{\prime}, \rho v^{\prime}\right)$ are proportional to the four differentials $(d x, d y, d u, d v)$.

5. The isoclinal surface elements. A surface element of $\Sigma_{3}$ is said to be an isocline if it is given by two equations of the form

$$
x^{\prime} / x_{1}^{\prime}=y^{\prime} / y_{1}^{\prime} \neq u^{\prime} / u_{1}^{\prime}=v^{\prime} v_{1}^{\prime} .
$$

Otherwise a surface element not an isocline is called a general surface element. There are $\infty^{2}$ isoclines among the $\infty^{4}$ surface elements of $\Sigma_{3}$.

There is a unique isocline through any given curve element. Therefore two isoclines never possess a common curve element.

By applying (6) to (7), we find that any isocline becomes an isocline under $G_{7}$. The group of correspondences between the isoclines is the six-parameter set

$$
\begin{aligned}
\mu X_{1}^{\prime}=a x_{1}^{\prime}+b y_{1}^{\prime}, & \nu U_{1}^{\prime}=\alpha u_{1}^{\prime}+\beta v_{1}^{\prime}, \\
\mu Y_{1}^{\prime}=c x_{1}^{\prime}+d y_{1}^{\prime}, & \nu V_{1}^{\prime}=\gamma u_{1}^{\prime}+\delta v_{1}^{\prime} .
\end{aligned}
$$

6. The invariants of two curve elements of $\Sigma_{3}$. In the first place, it is observed that the most general transformation of $G_{7}$ which will carry the curve element $(1,0,1,0)$ into the curve element $\left(\rho_{1} X_{1}^{\prime}\right.$, $\left.\rho_{1} Y_{1}^{\prime}, \rho_{1} U_{1}^{\prime}, \rho_{1} V_{1}^{\prime}\right)$ is of the form

$$
\begin{aligned}
\rho X^{\prime} & =\rho_{1} X_{1}^{\prime} x^{\prime}+b y^{\prime}, & \rho U^{\prime} & =\rho_{1} U_{1}^{\prime} u^{\prime}+\beta v^{\prime}, \\
\rho Y^{\prime} & =\rho_{1} Y_{1}^{\prime} x^{\prime}+d y^{\prime}, & \rho V^{\prime} & =\rho_{1} V_{1}^{\prime} u^{\prime}+\delta v^{\prime} .
\end{aligned}
$$

Under $G_{7}$, any curve element of $\Sigma_{3}$ may be carried into any other curve element of $\Sigma_{3}$. The canonical form of any curve element is $(1,0,1,0)$. There are $\infty^{4}$ such transformations.

A pair of curve elements is said to be an isoclinal pair if both elements lie in the same isocline. Otherwise it is said to be a general pair. The conditions necessary and sufficient for an isoclinal pair are (7).

Any curve element which lies in the same isocline with $(1,0,1,0)$ must be of the form $\left(x_{2}^{\prime}, 0, y_{2}^{\prime}, 0\right)$. The transform of this under the correspondence (9) is

$$
\begin{array}{ll}
\rho_{2} X_{2}^{\prime}=\rho_{1} X_{1}^{\prime} x_{2}^{\prime}, & \rho_{2} U_{2}^{\prime}=\rho_{1} U_{1}^{\prime} u_{2}^{\prime}, \\
\rho_{2} Y_{2}^{\prime}=\rho_{1} Y_{1}^{\prime} x_{2}^{\prime}, & \rho_{2} V_{2}^{\prime}=\rho_{1} V_{1}^{\prime} u_{2}^{\prime} .
\end{array}
$$


These equations immediately guarantee that the transformed element $\left(X_{2}^{\prime}, Y_{2}^{\prime}, U_{2}^{\prime}, V_{2}^{\prime}\right)$ is in the same isocline with $\left(X_{1}^{\prime}, Y_{1}^{\prime}, U_{1}^{\prime}, V_{1}^{\prime}\right)$. Moreover the expression

$$
X_{2}^{\prime} / X_{1}^{\prime} \cdot U_{1}^{\prime} / U_{2}^{\prime}=Y_{2}^{\prime} / Y_{1}^{\prime} \cdot V_{1}^{\prime} / V_{2}^{\prime}
$$

is invariant. By taking the logarithm of this invariant and then multiplying the result by $1 / 2 i$, it is found by (4) that the resulting invariant represents the angle between the isoclinal pair of curve elements.

Next let us observe that the group which preserves the curve element $(1,0,1,0)$ is

$$
\rho X^{\prime}=\rho_{1} x^{\prime}+b y^{\prime}, \rho Y^{\prime}=d y^{\prime}, \rho U^{\prime}=\rho_{1} u^{\prime}+\beta v^{\prime}, \rho V^{\prime}=\delta v^{\prime} .
$$

This group demonstrates that the curve element $\left(x^{\prime}, 0, u^{\prime}, 0\right)$ is preserved. Hence we find the following proposition to be true.

Theorem 1. An isoclinal pair of curve elements possesses the unique invariant

$$
\theta=\frac{1}{2 i} \log \frac{x_{2}^{\prime}}{x_{1}^{\prime}} \cdot \frac{u_{1}^{\prime}}{u_{2}^{\prime}}=\frac{1}{2 i} \log \frac{y_{2}^{\prime}}{y_{1}^{\prime}} \cdot \frac{v_{1}^{\prime}}{v_{2}^{\prime}} .
$$

This actually is the angle $\theta$ between the two directions.

The canonical forms of any isoclinal pair of curve elements are $(1,0,1,0)$ and $\left(x^{\prime}, 0, u^{\prime}, 0\right)$. There are $\infty^{4}$ such transformations.

Next any transformation of the group (12) which carries the curve element $(0,1,0,1)$ into the curve element $\left(\rho_{2} X_{2}^{\prime}, \rho_{2} Y_{2}^{\prime}, \rho_{2} U_{2}^{\prime}, \rho_{2} V_{2}^{\prime}\right)$ is of the form

$$
\begin{array}{ll}
\rho X^{\prime}=\rho_{1} x^{\prime}+\rho_{2} X_{2}^{\prime} y^{\prime}, & \rho U^{\prime}=\rho_{1} u^{\prime}+\rho_{2} U_{2}^{\prime} v^{\prime}, \\
\rho Y^{\prime}=\rho_{2} Y_{2}^{\prime} y^{\prime}, & \rho V^{\prime}=\rho_{2} V_{2}^{\prime} v^{\prime} .
\end{array}
$$

This demonstrates immediately that any general pair may be sent into any other general pair of curve elements.

The group which preserves the general pair of curve elements $(1,0,1,0)$ and $(0,1,0,1)$ is

$$
\rho X^{\prime}=\rho_{1} x^{\prime}, \quad \rho Y^{\prime}=\rho_{2} y^{\prime}, \quad \rho U^{\prime}=\rho_{1} u^{\prime}, \quad \rho V^{\prime}=\rho_{2} v^{\prime} .
$$

TheOREM 2. Any general pair of curve elements may be converted into any other general pair.

7. The nonexistence of invariants for an isocline paired with an isocline or a curve element. By (6), it may be proved that any isocline may be carried into the isocline $x^{\prime}=0, u^{\prime}=0$. The subgroup of (6) 
which preserves this isocline $x^{\prime}=0, u^{\prime}=0$ is, in curve element coordinates,

$$
\rho X^{\prime}=a x^{\prime}, \quad \rho Y^{\prime}=c x^{\prime}+d y^{\prime}, \quad \rho U^{\prime}=\alpha u^{\prime}, \quad \rho V^{\prime}=\gamma u^{\prime}+\delta v^{\prime} .
$$

Any isocline may be carried into any other isocline. The canonical form of an isocline is $x^{\prime}=0, u^{\prime}=0$. There are $\infty^{5}$ such transformations of $G_{7}$ which carry a given isocline into a prescribed isocline.

By (16) it may be proved that any isocline distinct from the isocline $x^{\prime}=0, u^{\prime}=0$ may be carried into the isocline $y^{\prime}=0, v^{\prime}=0$. The group which preserves the two isoclines $x^{\prime}=0, u^{\prime}=0$, and $y^{\prime}=0, v^{\prime}=0$ is

$$
\rho X^{\prime}=a x^{\prime}, \quad \rho Y^{\prime}=d y^{\prime}, \quad \rho U^{\prime}=\alpha u^{\prime}, \quad \rho V^{\prime}=\delta v^{\prime} .
$$

THEOREM 3. Any two distinct isoclines may be carried into any other two distinct isoclines.

The canonical forms of two distinct isoclines are $x^{\prime}=0, u^{\prime}=0$; and $y^{\prime}=0, v^{\prime}=0$. There are $\infty^{3}$ such transformations of $G_{7}$.

By combining (9) and (16), it is found that any curve element not on the isocline $x^{\prime}=0, u^{\prime}=0$ may be carried into the curve element $(1,0,1,0)$. The group preserving the isocline $x^{\prime}=0, u^{\prime}=0$ and the curve element $(1,0,1,0)$ is

$$
\rho X^{\prime}=\rho_{1} x^{\prime}, \quad \rho Y^{\prime}=d y^{\prime}, \quad \rho U^{\prime}=\rho_{1} u^{\prime}, \quad \rho V^{\prime}=\delta v^{\prime} .
$$

THEOREM 4. An isocline $\gamma$ and a curve element e not in $\gamma$ can be converted into any other isocline $\Gamma$ and a curve element $E$ not in $\Gamma$.

The canonical forms of an isocline $\gamma$ and a curve element $e$ not in $\gamma$ are $\gamma: u^{\prime}=0, x^{\prime}=0$; and $e(1,0,1,0)$. There are $\infty^{2}$ such transformations of $G_{7}$.

It may be proved that any isocline $\gamma$ and a curve element $e$ in $\gamma$ can be carried into the isocline $x^{\prime}=0, u^{\prime}=0$ and the curve element $(0,1,0,1)$. The group which preserves the isocline $x^{\prime}=0, u^{\prime}=0$ and the curve element $(0,1,0,1)$ is given by $(16)$.

An isocline $\gamma$ and a curve element $e$ in $\gamma$ can be carried into any other isocline $\Gamma$ and a curve element $E$ in $\Gamma$.

The canonical forms of an isocline $\gamma$ and a curve element $e$ in $\gamma$ are $\gamma: u^{\prime}=0, x^{\prime}=0$; and $e(0,1,0,1)$. There are $\infty^{4}$ such transformations.

8. The invariants for a hypersurface element paired with a curve element, or hypersurface element, or isocline. Any hypersurface element of $\Sigma_{3}$ is

$$
k x^{\prime}+l y^{\prime}+m u^{\prime}+n v^{\prime}=0 .
$$

Any hypersurface element may be given by $\pi(\sigma k, \sigma l, \sigma m, \sigma n)$. 
Note that $k$ and $l$, or $m$ and $n$, cannot both be zero as hypersurface elements which contain the two minimal planar directions $x^{\prime}=y^{\prime}=0$, or $u^{\prime}=v^{\prime}=0$ are excluded from consideration.

The induced group between the hypersurface elements of $\Sigma_{3}$ is

$$
\begin{aligned}
\sigma k & =a K+c L, & \sigma m & =\alpha M+\gamma N, \\
\sigma l & =b K+d L, & \sigma n & =\beta M+\delta N .
\end{aligned}
$$

Let a hypersurface element $\pi(k, l, m, n)$ and a curve element $e\left(x^{\prime}, y^{\prime}, u^{\prime}, v^{\prime}\right)$ be given, ( $e$ not in $\pi$ ). Upon drawing the isocline $\gamma$ through $e$, we find that $\gamma$ intersects $\pi$ in the curve element

$$
\begin{aligned}
& X^{\prime} / x^{\prime}=Y^{\prime} / y^{\prime}=-\omega\left(m u^{\prime}+n v^{\prime}\right), \\
& U^{\prime} / u^{\prime}=V^{\prime} / v^{\prime}=\omega\left(k x^{\prime}+l y^{\prime}\right) .
\end{aligned}
$$

By Theorem 1, we know that the angle between $e$ and the above curve element is invariant.

By (12) and (20), we see that the group which preserves the curve element $(1,0,1,0)$ may be written in hypersurface element coordinates as

$$
\sigma k=\rho_{1} K, \quad \sigma l=b K+d L, \quad \sigma m=\rho_{1} M, \quad \sigma n=\beta M+\delta N .
$$

By these equations, it may easily be proved that the hypersurface element $\pi(\sigma k, \sigma l, \sigma m, \sigma n)$ can be sent into the hypersurface element $\Pi(K, 0, M, 0)$. The group which preserves the curve element $e(1,0,1,0)$ and the hypersurface element $\pi(k, 0, m, 0)$ is

$$
\sigma k=\rho_{1} K, \quad \sigma l=d L, \quad \sigma m=\rho_{1} M, \quad \sigma n=\delta N .
$$

TheOREM 5. A hypersurface element $\pi$ and a curve element $e$ (e not in $\pi$ ) possess the unique invariant

$$
\theta=\frac{1}{2 i} \log \left[-\frac{m u^{\prime}+n v^{\prime}}{k x^{\prime}+l y^{\prime}}\right] .
$$

This is the angle between $e$ and the unique curve element $e^{*}$ in $\pi$ such that $e$ and $e^{*}$ form an isoclinal pair. This is identical with Kasner's pseudoangle.

The canonical forms of a hypersurface element $\pi$ and a curve element $e(e \operatorname{not}$ in $\pi)$ are $\pi: k x^{\prime}+m u^{\prime}=0$ and $e(1,0,1,0)$. The pseudoangle is then $(1 / 2 i) \log (-m / k)$. There are $\infty^{2}$ such transformations.

Any hypersurface element contains a unique isocline. Thus the isocline in the hypersurface element $\pi(k, l, m, n)$ is

$$
k x^{\prime}+l y^{\prime}=0, \quad m u^{\prime}+n v^{\prime}=0 .
$$


A hypersurface element $\pi$ and a curve element $e$ contained in $\pi$ but not in the isocline of $\pi$ can be carried into any other hypersurface element $\Pi$ and a curve element $E$ contained in $\Pi$ but not in the isocline of II.

The canonical forms of a hypersurface element $\pi$ and a curve element $e$ contained in $\pi$ but not in the isocline of $\pi$ are $\pi: x^{\prime}=u^{\prime}$ and $e(1,0,1,0)$. There are $\infty^{2}$ such transformations.

A hypersurface element $\pi$ and a curve element $e$ contained in the isocline of $\pi$ can be sent into any other hypersurface element $I$ and a curve element $E$ contained in the isocline of $\Pi$.

The canonical forms of a hypersurface element $\pi$ and a curve element $e$ contained in the isocline of $\pi$ are $\pi: y^{\prime}+v^{\prime}=0$ and $e(1,0,1,0)$. There are $\infty^{3}$ such transformations.

A pair of hypersurface elements are said to be an isoclinal pair if they intersect in an isocline. Otherwise they are said to be a general pair.

In hypersurface element coordinates, the equations of an isocline are

$$
k / k_{1}=l / l_{1} \neq m / m_{1}=n / n_{1} .
$$

Of course, these are the conditions that the hypersurface elements $\left(k_{1}, l_{1}, m_{1}, n_{1}\right)$ and $(k, l, m, n)$ form an isoclinal pair.

THEOREM 6. An isoclinal pair of hypersurface elements $\pi_{1}$ and $\pi_{2}$ possess the fundamental invariant

$$
\theta=\frac{1}{2 i} \log \frac{k_{1}}{k_{2}} \cdot \frac{m_{2}}{m_{1}}=\frac{1}{2 i} \log \frac{l_{1}}{l_{2}} \cdot \frac{n_{2}}{n_{1}} .
$$

This is Kasner's pseudo-angle between $\pi_{2}$ and any curve element of $\pi_{1}$.

The canonical forms of an isoclinal pair of hypersurface elements are $x^{\prime}+u^{\prime}=0$ and $k x^{\prime}+m u^{\prime}=0$. The invariant (27) is then $(1 / 2 i) \log (m / k)$. There are $\infty^{4}$ such transformations.

THEOREM 7. In the other possible pairs of elements, no further invariants are found.

On examination, we find the following standard forms:

The canonical forms of a general pair of hypersurface elements are $x^{\prime}+u^{\prime}=0$ and $y^{\prime}+v^{\prime}=0$. There are $\infty^{1}$ such transformations.

The canonical forms of any isocline $\gamma$ and a hypersurface element $\pi, \gamma$ not in $\pi$, are $\gamma: y^{\prime}=0, v^{\prime}=0$, and $\pi: x^{\prime}+u^{\prime}=0$. There are $\infty^{2}$ such transformations.

The canonical forms of an isocline $\gamma$ and a hypersurface element 
$\pi, \gamma$ in $\pi$, are $\gamma: y^{\prime}=0, v^{\prime}=0$, and $\pi: y^{\prime}+v^{\prime}=0$. There are $\infty^{4}$ such transformations.

9. The invariants for a general surface element paired with a curve element, or a hypersurface element, or a general surface element, or isocline. A surface element, not an isocline, may be given by $u^{\prime}$ and $v^{\prime}$ expressed as linear functions of $x^{\prime}$ and $y^{\prime}$; or by $x^{\prime}$ and $y^{\prime}$ expressed as linear functions of $u^{\prime}$ and $v^{\prime}$. Because of the symmetry of our group (6), we find that it always can be supposed that a general surface element can be written in the form

$$
u^{\prime}=p x^{\prime}+q y^{\prime}, \quad v^{\prime}=r x^{\prime}+s y^{\prime} .
$$

Since we wish to omit from consideration those general surface elements, which contain a curve element of the minimal planar directions $x^{\prime}=y^{\prime}=0$, or $u^{\prime}=v^{\prime}=0$, we find that $p s-q r \neq 0$.

The correspondence between the general surface elements is

$$
\begin{aligned}
& (\alpha \delta-\beta \gamma) p=a \delta P+c \delta Q-a \beta R-c \beta S, \\
& (\alpha \delta-\beta \gamma) q=b \delta P+d \delta Q-b \beta R-d \beta S, \\
& (\alpha \delta-\beta \gamma) r=-a \gamma P-c \gamma Q+a \alpha R+c \alpha S, \\
& (\alpha \delta-\beta \gamma) s=-b \gamma P-d \gamma Q+b \alpha R+d \alpha S .
\end{aligned}
$$

Let $S(p, q, r, s)$ be a given general surface element. Through each curve element of $S$, there is a unique isocline. There are, therefore, $\infty^{1}$ isoclines which intersect a given surface element $S$. These isoclines generate a nondegenerate quadric regulus $R$, which is given by

$$
\frac{u^{\prime}}{v^{\prime}}=\frac{p x^{\prime}+q y^{\prime}}{r x^{\prime}+s y^{\prime}} \text {. }
$$

Let $e\left(x^{\prime}, y^{\prime}, u^{\prime}, v^{\prime}\right)$ be a curve element of the regulus $R$ but not in $S$. The isocline through $e$ will intersect the general surface element $S$ in the curve element $e^{*}$ given by

$$
X^{\prime} / x^{\prime}=Y^{\prime} / y^{\prime}=\omega u^{\prime}, \quad U^{\prime} / u^{\prime}=V^{\prime} / v^{\prime}=\omega\left(p x^{\prime}+q y^{\prime}\right) .
$$

By Theorem 1, the angle between $e$ and $e^{*}$ is invariant.

Since any general pair of curve elements may be carried into any other general pair, it follows that any general surface element may be sent into any other general surface element. In particular, we may carry any general surface element into the general surface element $u^{\prime}=x^{\prime}, v^{\prime}=y^{\prime}$.

Let us find the subgroup of $G_{7}$ which leaves the general surface element $u^{\prime}=x^{\prime}, v^{\prime}=y^{\prime}$ invariant. By (29), we must have 


$$
\alpha \delta-\beta \gamma=a \delta-c \beta=-b \gamma+d \alpha, \quad 0=b \delta-d \beta=-a \gamma+c \alpha .
$$

By these equations, it follows immediately that $\alpha=a, \beta=b, \gamma=c$, $\delta=d$. Thus the subset of $G_{7}$, which leaves our general surface element $u^{\prime}=x^{\prime}, v^{\prime}=y^{\prime}$ fixed is the three-parameter group in curve element coordinates

$$
\rho X^{\prime}=a x^{\prime}+b y^{\prime}, \quad \rho U^{\prime}=a u^{\prime}+b v^{\prime}, \quad \rho Y^{\prime}=c x^{\prime}+d y^{\prime}, \quad \rho V^{\prime}=c u^{\prime}+d v^{\prime} .
$$

Now there exists a transformation of $G_{7}$ which carries our general surface element into $S: u^{\prime}=x^{\prime}, v^{\prime}=y^{\prime}$. Therefore our curve element is carried into a curve element which belongs to the regulus of $S$. Hence the coordinates of this new curve element satisfy the condition $u^{\prime} / v^{\prime}=x^{\prime} / y^{\prime}$. Now in (33) take $-c / d=y^{\prime} / x^{\prime}=v^{\prime} / u^{\prime}$. Obviously this transformation will take our curve element into the curve element $\left(x^{\prime}, 0, u^{\prime}, 0\right)$.

TheOREM 8. Let the curve element $e\left(x^{\prime}, y^{\prime}, u^{\prime}, v^{\prime}\right)$ be on the regulus $R$ of the general surface element $S: u^{\prime}=p x^{\prime}+q y^{\prime}, v^{\prime}=r x^{\prime}+s y^{\prime}$. That is, let $\left(u^{\prime} / v^{\prime}\right)=\left(p x^{\prime}+q y^{\prime}\right) /\left(r x^{\prime}+s y^{\prime}\right)$. Then $e$ and $S$ possess the unique invariant

$$
\theta=\frac{1}{2 i} \log \frac{u^{\prime}}{p x^{\prime}+q y^{\prime}}=\frac{1}{2 i} \log \frac{v^{\prime}}{r x^{\prime}+s y^{\prime}} .
$$

This is the angle between $e$ and the curve element $e^{*}$ on $S$ such that e and $e^{*}$ are an isoclinal pair.

The canonical forms of a curve element $e$ and a general surface element $S, e$ on the regulus $R$ of $S$ but not in $S$, are $e\left(x^{\prime}, 0, u^{\prime}, 0\right)$ and $S: u^{\prime}=x^{\prime}, v^{\prime}=y^{\prime}$. There are $\infty^{2}$ such transformations. The invariant (34) is then $\theta=(1 / 2 i) \log \left(u^{\prime} / x^{\prime}\right)$.

Any pair consisting of a general surface element $S$ and a curve element $e$ contained in $S$ can be converted into any other such pair.

The canonical forms of a general surface element $S$ and a curve element $e$ contained in $S$ are $S: u^{\prime}=x^{\prime}, v^{\prime}=y^{\prime}$ and $e(1,0,1,0)$.

Next let us note that the transformation (33) where

$$
\begin{array}{ll}
a=\frac{\rho_{1}}{2 i}\left(X_{1}^{\prime}-U_{1}^{\prime}\right), & b=\frac{\rho_{1}}{2}\left(X_{1}^{\prime}+U_{1}^{\prime}\right), \\
c=\frac{\rho_{1}}{2 i}\left(Y_{1}^{\prime}-V_{1}^{\prime}\right), & d=\frac{\rho_{1}}{2}\left(Y_{1}^{\prime}+V_{1}^{\prime}\right),
\end{array}
$$

will carry the curve element $(i, 1,-i, 1)$, not in the regulus of the general surface element $S: u^{\prime}=x^{\prime}, v^{\prime}=y^{\prime}$, into any curve element $\left(\rho_{1} X_{1}^{\prime}, \rho_{1} Y_{1}^{\prime}, \rho_{1} U_{1}^{\prime}, \rho_{1} V_{1}^{\prime}\right)$, not in the regulus of $S$. 
We also note that the only transformation which preserves the general surface element $u^{\prime}=x^{\prime}, v^{\prime}=y^{\prime}$ and the curve element $(i, 1,-i, 1)$ is the identity.

THEOREM 9. There exist no invariants between any curve element $e$ and a general surface element $S$ such that $e$ is not on the regulus $R$ of $S$. There exists one and only one transformation of $G_{7}$ which will carry any such general pair into another general pair.

Let $\left(x^{\prime}, y^{\prime}, u^{\prime}, v^{\prime}\right)$ be the coordinates of the curve element of contact between the tangent hypersurface $\pi(k, l, m, n)$ and the regulus $R$ of the general surface element $S$. Then

(36) $\rho k=r u^{\prime}-p v^{\prime}, \rho l=s u^{\prime}-q v^{\prime}, \rho m=r x^{\prime}+s y^{\prime}, \rho n=-p x^{\prime}-q y^{\prime}$.

Solving these equations for $\left(x^{\prime}, y^{\prime}, u^{\prime}, v^{\prime}\right)$, we find

$$
\sigma x^{\prime}=-m q-n s, \quad \sigma u^{\prime}=p l-k q, \quad \sigma y^{\prime}=p m+r n, \quad \sigma v^{\prime}=-k s+r l .
$$

The angle between this curve element $e$ and the curve element $e^{*}$ in $S$ such that $e$ and $e^{*}$ are an isoclinal pair is invariant. The preceding equations in conjunction with Theorem 8 yield the following information.

THEOREM 10. Let a hypersurface element $\pi(k, l, m, n)$ be tangent to the regulus $R$ of the general surface element $S: u^{\prime}=p x^{\prime}+q y^{\prime}$, $v^{\prime}=r x^{\prime}+s y^{\prime}$. The condition necessary and sufficient for this is $(-n / m)$ $=(k q-p l) /(k s-r l)$. Then $\pi$ and $S$ possess the unique invariant

$$
\theta=\frac{1}{2 i} \log \left[\frac{q k-p l}{n(p s-q r)}\right]=\frac{1}{2 i} \log \left[-\frac{s k-r l}{m(p s-q r)}\right] .
$$

This is the angle between the curve element e of contact between the hypersurface element $\pi$ and the regulus $R$, and the curve element $e^{*}$ on the general surface element $S$ such that $e$ and $e^{*}$ form an isoclinal pair.

Again by Theorem 9 and equations (37), we deduce the following theorem:

THEOREM 11. There exist no invariants between a hypersurface element $\pi$ and a general surface element $S$ with $\pi$ not tangent to the regulus $R$ of $S$. There exists one and only one transformation of $G_{7}$ which will carry any such general pair into another general pair.

The canonical forms are $S: u^{\prime}=x^{\prime}, v^{\prime}=y^{\prime}$, and $\pi: i x^{\prime}+y^{\prime}-i u^{\prime}+v^{\prime}$ $=0$.

Let $\theta$ be the angle between any curve element $e$ of the regulus $R_{1}$ of the general surface element $S_{1}$ and the curve element $e^{*}$ on $S_{1}$ such 
that $e$ and $e^{*}$ are an isoclinal pair. Upon setting $\phi=e^{2 i \theta}$, we find by Theorem 11 that the regulus $R_{1}$ may be given parametrically by

$$
u^{\prime}=\phi\left(p_{1} x^{\prime}+q_{1} y^{\prime}\right), \quad v^{\prime}=\phi\left(r_{1} x^{\prime}+s_{1} y^{\prime}\right) .
$$

Now the general surface element $S_{2}$ will intersect $R_{1}$ in two curve elements $e_{i}$ and $e_{j}$, given by

$$
\begin{aligned}
& u^{\prime}=\phi\left(p_{1} x^{\prime}+q_{1} y^{\prime}\right)=p_{2} x^{\prime}+q_{2} y^{\prime}, \\
& v^{\prime}=\phi\left(r_{1} x^{\prime}+s_{1} y^{\prime}\right)=r_{2} x^{\prime}+s_{2} y^{\prime} .
\end{aligned}
$$

The elimination of $x^{\prime}$ and $y^{\prime}$ gives a quadratic equation in $\phi$. The roots of this quadratic equation are therefore invariants of the two general surface elements $S_{1}$ and $S_{2}$.

Now we shall show that these two invariants are the only ones. In the first place, we may assume that $S_{1}$ is of the form $u^{\prime}=x^{\prime}, v^{\prime}=y^{\prime}$. Then from (40), we find that our invariants are the roots of

$$
\phi^{2}-\left(p_{2}+s_{2}\right) \phi+\left(p_{2} s_{2}-r_{2} q_{2}\right)=0 .
$$

Since the sum and product of these roots are invariant, we know that $\left(p_{2}+s_{2}\right)$ and $\left(p_{2} s_{2}-r_{2} q_{2}\right)$ are both invariants.

Next we shall show that we can carry our surface $S_{2}\left(p_{2}, q_{2}, r_{2}, s_{2}\right)$ into $\left(P_{2}, 0,0, S_{2}\right)$. In the first place, the two invariants of the preceding paragraph yield the following two conditions

$$
P_{2}+S_{2}=p_{2}+s_{2}, \quad P_{2} S_{2}=p_{2} s_{2}-r_{2} q_{2} .
$$

The elimination of $s_{2}$ yields the condition

$$
p_{2}^{2}-\left(P_{2}+S_{2}\right) p_{2}+P_{2} S_{2}+r_{2} q_{2}=0 .
$$

Let us in the first place assume that our general surface element $S_{2}$ is contained in the regulus $R_{1}$ of $S_{1}$. Then $S_{2}$ must be of the form $\left(p_{2}, 0,0, p_{2}\right)$ which is the desired form. As a matter of fact, it is seen that our group (33) preserves any general surface element $S_{2}$ contained in $R_{1}$.

Now let us suppose that our general surface element $S_{2}$ is not contained in the regulus $R_{1}$. Then its transformed surface element $\left(P_{2}, 0,0, S_{2}\right)$ is not contained in $R_{1}$ so that $P_{2} \neq S_{2}$.

The group (33) shows that if $\left(p_{2}, q_{2}, r_{2}, s_{2}\right)$ is carried into $\left(P_{2}, 0,0, S_{2}\right)$, then

$$
\begin{aligned}
& (a d-b c) p_{2}=a d P_{2}-b c S_{2}, \quad(a d-b c) r_{2}=-a c\left(P_{2}-S_{2}\right), \\
& (a d-b c) q_{2}=b d\left(P_{2}-S_{2}\right), \quad(a d-b c) s_{2}=-b c P_{2}+a d S_{2} .
\end{aligned}
$$

By use of the first of equations (42), it may be shown that the last of 
the preceding equations is equivalent to the first equation. Hence our conditions reduce to

$$
\begin{aligned}
a d\left(p_{2}-P_{2}\right) & =b c\left(p_{2}-S_{2}\right), \\
(a d-b c) q_{2} & =b d\left(P_{2}-S_{2}\right), \\
(a d-b c) r_{2} & =-a c\left(P_{2}-S_{2}\right) .
\end{aligned}
$$

Now in the first of these equations, we see that $p_{2}-P_{2}$ and $p_{2}-S_{2}$ cannot both be zero, for otherwise $P_{2}=S_{2}$, which is impossible. Hence there exists a nonzero number $\lambda$ so that

$$
a d=\lambda\left(p_{2}-S_{2}\right), \quad a c=-\lambda r_{2}, \quad b c=\lambda\left(p_{2}-P_{2}\right), \quad b d=\lambda q_{2} .
$$

From these equations, we deduce that since $\lambda \neq 0$, then

$$
\frac{c}{d}=-\frac{r_{2}}{p_{2}-S_{2}}=\frac{p_{2}-P_{2}}{q_{2}}
$$

Of course the last equation is true because of (43). Substituting (47) into (46), we see that $a: b: c: d$ are determined except for an arbitrary constant.

Theorem 12. Two general surface elements $S_{1}$ and $S_{2}$ possess the two differential invariants $\phi_{1}$ and $\phi_{2}$ which are roots of the equation

$$
\left(p_{1} s_{1}-q_{1} r_{1}\right) \phi^{2}+\left(q_{1} r_{2}+q_{2} r_{1}-p_{1} s_{2}-p_{2} s_{1}\right) \phi+\left(p_{2} s_{2}-q_{2} r_{2}\right)=0 \text {. }
$$

If we let $\theta=(1 / 2 i) \log \phi$, then $\theta$ is actually the angle between the curve element $e_{i}$, or $e_{j}$, in which $S_{2}$ intersects the regulus $R_{1}$ of $S_{1}$ and the curve element $e^{*}$ in $S_{1}$ such that $e_{i}$, or $e_{j}$, and $e^{*}$ are an isoclinal pair. There are no more invariants.

Finally, we may prove the following result.

THEOREM 12*. There are no invariants between a general surface element $S$ and an isocline $\gamma$. Any such pair may be carried into any other.

In conclusion, we observe that Kasner's pseudo-angle may quite easily be extended to characterize the group of pseudo-conformal transformations defined by $n$ complex functions of $n$ complex variables. This group is characterized within the group of arbitrary point transformations of a $2 n$-dimensional space by the preservation of the pseudo-angle between a curve and a $(2 n-1)$-dimensional hypersurface at their common point of intersection.

Columbia University and

Illinois Institute of TeChNOLOGY 